\title{
Construction and Application of Information Base of Urban Residential Landscape Based on Big Data
}

\author{
Wang Shunhui ${ }^{1 \mathrm{a}}$ and Zou Jie $\mathrm{e}^{2 \mathrm{~b}}$ \\ 1. Institute of Arts and Technology,Harbin University of Science and \\ Technology, China \\ 2.College of Foreign Languages, Harbin University of Science and \\ Technology, China \\ a.Wangshunhui@yahoo.com,b.en.landscape@gmail.com
}

\begin{abstract}
Big Data era is changing people's way of thinking and working, urban residential landscape industry needs to absorb the production methods development outcomes of big data techniques. People need to build landscape environment like which more suitable for their material and spiritual life based on big data. In this paper, the urban residential landscape information database system architecture has been designed, focusing on the data source analysis, conceptual design, logical design and physical design. And further to the database techniques, such as data dictionary, ER model, SQL, and Web publishing platform, network applications techniques were introduced. Development and application of urban residential landscape database is a significant way to expand urban landscape environment construction.
\end{abstract}

Keywords: Urban residential environment, Environmental landscape, Database, SQL, Dreamweaver

\section{Introduction}

Big Data techniques refers to the amount of data is too large to capture, manage, process and defragment in order to become to the useful message for Business Decision of Enterprise, by the current mainstream software tools in a reasonable time.[1]Big Data has 4V features: Volume, Velocity, Variety, value .Research institute Gartner defined "Big Data" as massive, high-growth and diversified Information Assets, which needs new processing mode to have stronger decision-making power, strength, insight discovery and process optimization ability.Paper research on application techniques in the field of residential landscape construction, and providing some experiences and suggestions.

\subsection{Techniques of Database}

Gartner believes that big data is beyond the commonly used hardware environment and software tools in an acceptable time for its users collect, manage, and the ability to process the data.[2] For is not implemented, including urban residential landscape, most of the industry informatization, the database construction of the industry, is a starting point for big data, and industry in the future development of inevitable direction. Database can be used for the construction of MY SQL, MS SQL and DB2, release, connected with the retrieval application can use the DW, etc. Product or technique. Data processing part consists of data acquisition, data sorting, statistical analysis and the professional classification processing, etc., through statistical analysis and professional classification processing of residential landscape information, in the face of the different needs of users with the key key retrieval use etc. Residential landscape of the ability of information retrieval and information use, is the measure of a city construction informationization 
ability and innovative consciousness of the key factors. DeamWeaver can make Php + MySQL Web site, but don't know the Php and MySQL application of language, only use the dreamweaver, functional limitations, built in dreamweaver platform site, easy to manage, connect to the database, to set up the data sets, just simple dynamic pages using dreamweaver, need more complex SQL technical support can be realized. So this article recommend the important technique of Php and MySQL and the relevant technique of DW.

Established for big data era driven by database, used in the Web site to solve the problem of urban residential landscape construction, management and application, make it organized highly effective work, is the necessary way of contemporary living environment and urban landscape construction.[2] The application of the database structure is:

1.Using MySQL database technique design to create urban residential landscape, to create all kinds of related tables in a database, and according to user's need to get the corresponding information.

2.The use of Php variables, conditions, and control technique such as the structure, the write access to residential database and provide Html scripts to the user's browser.

3.The DW visualization interface technique, constructing the server, and the test and so on.

\subsection{Features of Urban Residential Landscape Data}

With the development of China's urbanization, especially the new development of urbanization in 2013, urban residential landscape becomes an important contemporary issue, an important form of peoples living environment. Mainly displays in the new commodity housing development, the existing residential area landscape environment, urban residential community square landscape, and residential streets landscape. In the Age of Big Data, the construction of urban residential landscape can't do without data. A large number of residential environment landscape information is important condition and tool to improving the level of residential environment landscape design.

This study is an interdisciplinary research and practice combined with the major of landscape design and Computer Data Processing, in order to develop a database of contemporary urban residential landscape by SQL techniques, point out the value and significance of putting the database into network application by DW techniques. In the Age of Big Data, Information is the key to winning. The way to get information is scattered, less systematic, and uncomprehensive.[3] It is a task of top priority to create a database of urban residential landscape. The basic objects stored in the database is organized, shareable data set, including images, text, sound, graphics. To create a database of urban residential landscape, and to put it into network application by DW techniques, is an important part of Intelligent residential.

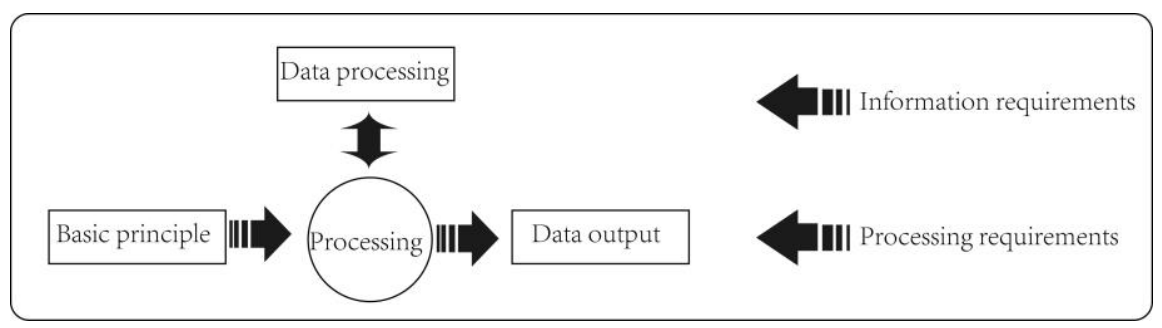

Figure 1. Model of Database Basic Principle 
The database is divided into needs analysis, structural design, physical design, implementation and maintenance aspects. Needs analysis is to research the target object, clarify the user' $s$ requirements, Identify the system functionality. It is necessary to give enough thought to the user' $\mathrm{s}$ change. The main problem in this stage is the user' $s$ information, safety and integrity in the process. On the one hand, because users do not understand the computer related techniques, so there is a certain difficulty in the process of demand expression. On the other hand, the designers of the database do not the major of the users. in the professional information collection and information processing, it' $s$ difficult to understand the users' $s$ true requirements, even misunderstand users' s requirements. All of the above will will eventually lead to mismatch between the system and user, and even a lot of waste of human and material resources.

On the basis of survey and collection of our residential landscape resources, crease a database of China' s urban residential landscape, and Information management system. The system not only collected the general characteristics of the residential landscape but also joined the ornamental characteristics of the landscape, garden mood, commonly used index, the market reference price, morphological pictures, photographs, and other applications. The system uses a flexible query, which greatly facilitates the users to use, and information and standardized management systems residential landscape.

\section{Architecture and Design of the Urban Residential Landscape Database System}

Domestic with high value and the typical residential landscape resources, collect built into a comprehensive information database, the use of modern information technique to develop the management information database. The system should have data entry, query, statistic and analysis, image browsing, system maintenance and other major function, should have comprehensive functions, easy operation, large amount of information data, fast data processing, data output characteristics of flexible system for related industries engaged in environmental planning and landscape design research, decision-making, teaching, production, management and other departments of the user, provides an existing residential area landscape resources of our country, more systematic and comprehensive application of the database. [4]The system architecture of the library should include data analysis, concept structure design, logic design, physical design and implementation of maintenance section.

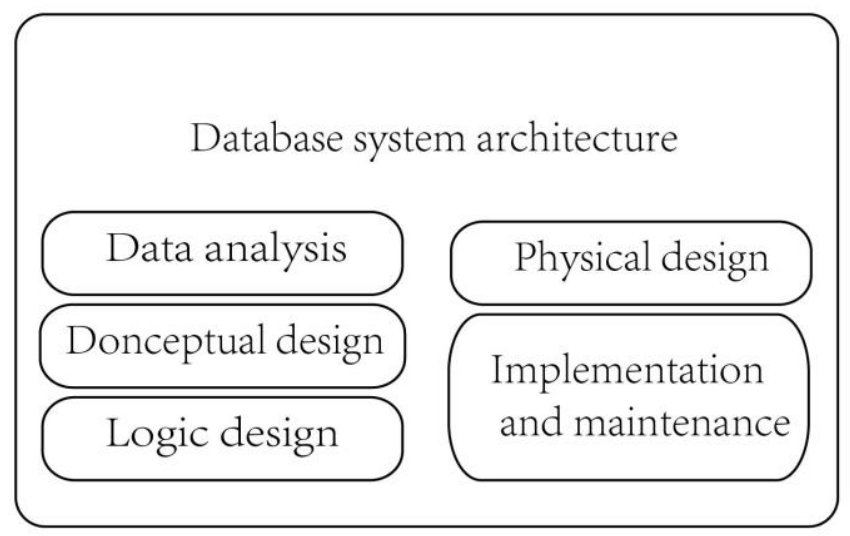

Figure 2. Urban Residential Landscape Database Architecture 


\subsection{Data Analysis}

Urban residential landscape has a large amount of data and many kinds of monitoring and sampling, such as complicated structure specific. Analysis from the data form, including quantitative numeric data and non numeric text, graphics, images, audio, video, and hypertext; The schedule was obtained from the data point of view, including online collection, manual record, dynamic monitoring, etc., as well as the various forms of historical data; From the connotation of the data, including direct first-hand original data, integrate data through sorting calculation, and through professional statistical analysis results of data processing.

Online acquisition refers to through the network retrieval, has certain influence on the regional characteristics of building residential landscape of the electronic data collection behavior, including landscape scene photos, aerial map form; Manual record refers to the scene photos, video recording, written records and draw pictures; Dynamic monitoring mainly for landscape growing principle, for the same project in spring, summer, autumn, winter of different seasons and different years of plant scale, shape and color changes, and the change and the environment construction, water, facilities and other environmental elements, constitute the integrated landscape dynamic monitor and record.

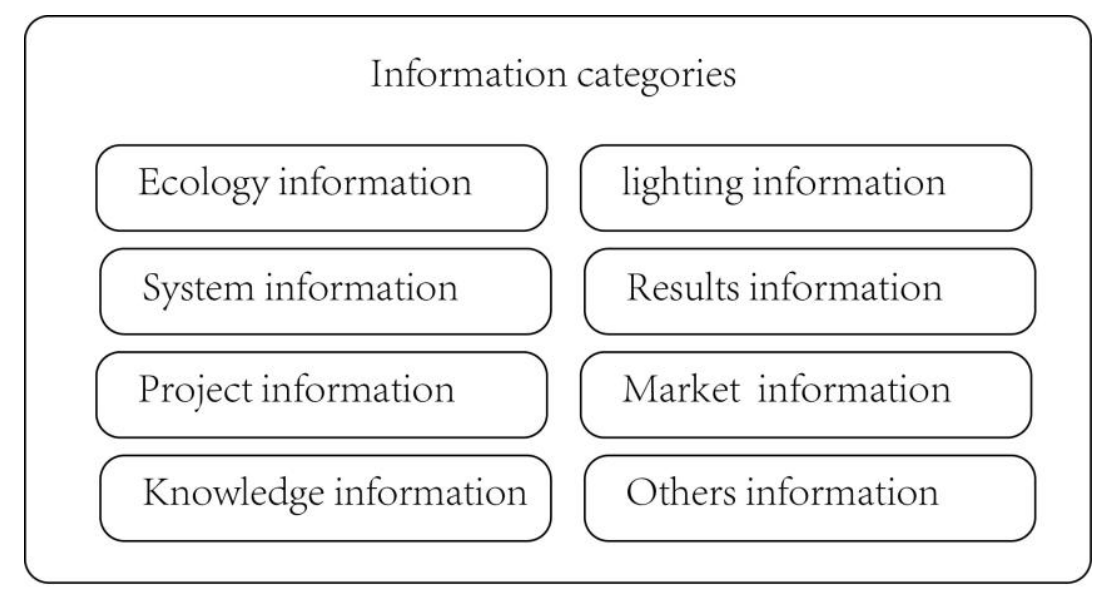

Figure 3. Model of Information Categories

To meet the demand of system operation, the user needs, in many aspects as well as the system completeness residential landscape storage of database need more information, such as system information, project information, project information, knowledge, information, ecology, lighting information, results, information, market information, etc. Information system is the system operation and management level of information, including user information, information management, control and operation log, etc.; Background information of the project including project development, investment, information architects, use the crowd analysis, project overall composition, such as engineering information including the construction of the base profile, landscape planning and design, information, operation information, all kinds of parameters, drawings, photographs, documents, reports, meeting audio and video data, etc.; Information includes knowledge needed by the business logic of knowledge, including basic public information, laws and regulations, technical data, models, principles, expert knowledge and so on; Results information including statistics, analysis and evaluation, the conclusion, decisionmaking Suggestions and feedback information, etc.; Market information including the price, the market feedback information, user evaluation, etc.; Ecological information 
including plant species, plant shape, climate, hydrology, soil and all kinds of common animals.

\subsection{Conceptual Design}

The bottom of the urban residential landscape database, should set up a comprehensive residential landscape project based database, for data input, data management, data analysis and results published each subsystem such as support.

For data input subsystem, should realize automatic acquisition, artificial records, dynamic monitoring, and the combination of historical load data. By automatically collect data interface, will realize the monitoring data of the automation system in comprehensive database, or by scanning way of dealing with the mobile data acquisition device files, online input text, audio and video and so on historical data. [5]Data input should possess efficacy, by setting the BGF calculation formula, automatic to convert the BGF, gross error elimination, error repair, and other functions, to ensure the reliability of the incoming information. Implementing method according to specific needs, select the server trigger technique or application programming processing.

For data management subsystem of the application, the function is based on the database, should provide convenient and flexible information about project, landscape grade, actual photos, residents participation, place to experience the empirical aspects of query function, safe and reliable data add, delete, modify and improve the output of the reference functions, production specification requirements or user customization of all kinds of files, including professional report, professional drawing graphics, audio and video media information, etc. In addition, the database should have strong function of statistics, analysis and summary. Above need not only between application and database interface, rapid and reliable data and need the data itself is concise, complete data structure.

For data analysis subsystem, the database should be to provide professional model analysis, comprehensive analysis of online and offline analysis, expert consultation and so on model and knowledge required for the user's needs as well as the relevant parameters. These data should be open to the users can query maintenance and expansion. Also need to have intermediate information storage function, database for the exchange of data between applications and sharing, etc.

Published subsystem should have the information such as monitoring results, analysis and expert advice to the function of the integrated management, and through the Web is released to the world. The database and Web server interface is put forward the corresponding requirements.

The concept of database structure is logical design, on the physical implementation, all data should be unified as far as possible in one or a few actual database, for data for designing and operating data correlation relationship. For system function, the different needs of users, the parts can use view technique now users are relatively independent and complete classification (professional) database.

\subsection{Logic Design}

The database logical design is the core of the relational database system optimization design. Logic design is mainly for database tables, as well as the relationship between the table and table design. Good logic design is the foundation of database and application performance optimization. Logical database design stage should be as follows: to complete the task to create data dictionary, E-R model, defining the standard object naming conventions, tables, fields, index, the design of the primary and foreign keys, etc. 
Data dictionary is a data set, it with data in the database basic unit for the unit, according to certain order, and make detailed instructions on its content. Data dictionary for the meaning of all the data elements and structure, type, size, format, unit of measure, accuracy and threshold regulation, to ensure that the developers use consistent data definition. Data dictionary information about the system is the system design, system implementation and system maintenance basis, as the main task of the logical design, data dictionary not only in the process of data analysis and design has the very important role, and is of great significance in the implementation phase.[6]

Urban residential landscape for the table metadata database dictionary table (table 1) and column metadata table (table 2). According to user needs the database table structure according to the metadata table design is good, use the written application can quickly create a database table content can be converted to SQL statements, so as to establish a database. With some engineering database integrate data table, for example, data dictionary and SQL statements are presented.

Table 1. Metadata Table

\begin{tabular}{|c|c|c|c|c|c|c|}
\hline Field name & Length of type & Null value & Meaning & Main code & Outer code & Instructions \\
\hline Table number & CHAR & NOTNULL & & $\checkmark$ & & \\
\hline Table type & CHAR & NOTNULL & & & & \\
\hline English table name & VARCHAR & NOTNULL & & & & \\
\hline Chinese table name & VARCHAR & NOTNULL & & & & \\
\hline
\end{tabular}

Metadata can table in the database query system, choose any query column, according to the results show that the way and the way of ordering convenient combination query.

Table 2. Column Metadata

\begin{tabular}{|c|c|c|c|c|c|c|}
\hline Field name & Length of type & Null value & Meaning & Main code & Outer code & Instructions \\
\hline Table number & CHAR & NOTNULL & & $\checkmark$ & $\checkmark$ & \\
\hline Table type & CHAR & NOTNULL. & & $\checkmark$ & & \\
\hline English table name & VARCHAR & NOTNULLL & & & & \\
\hline Chinese table name & e VARCHAR & NOTNULL. & & & & \\
\hline Column type & VARCHAR & NOTNULLL & & & & \\
\hline Column width & CHAR & NOTNULL. & & & & \\
\hline Unit & CHAR & NULL & & & & \\
\hline Primary key & CHAR & NOTNULL. & & & & \\
\hline Null value & CHAR & NOTNULL & & & & \\
\hline Value range & VARCHAR & NULL & & & & \\
\hline Note & VARCHAR & NULLL & & & & \\
\hline
\end{tabular}

Build table SQL statement is as follows:

The create table dbo. XLDED_SA (PN char (1) not null,

DATE_TIME smalldatetime not null,

DSPLC numeric $(7,2)$ null,

Note char (1) the null,

Note1 char (1) the null,

The constraint XLDED_SA_x PRIMARY KEY CLUSTERED (PN, DATE_TIME))

To the design of the other tables and fields on the basis of the above methods, the database logic design should consider the integrity of the data, need to rely on the rules, foreign keys, cascade triggers, stored procedures, etc. 


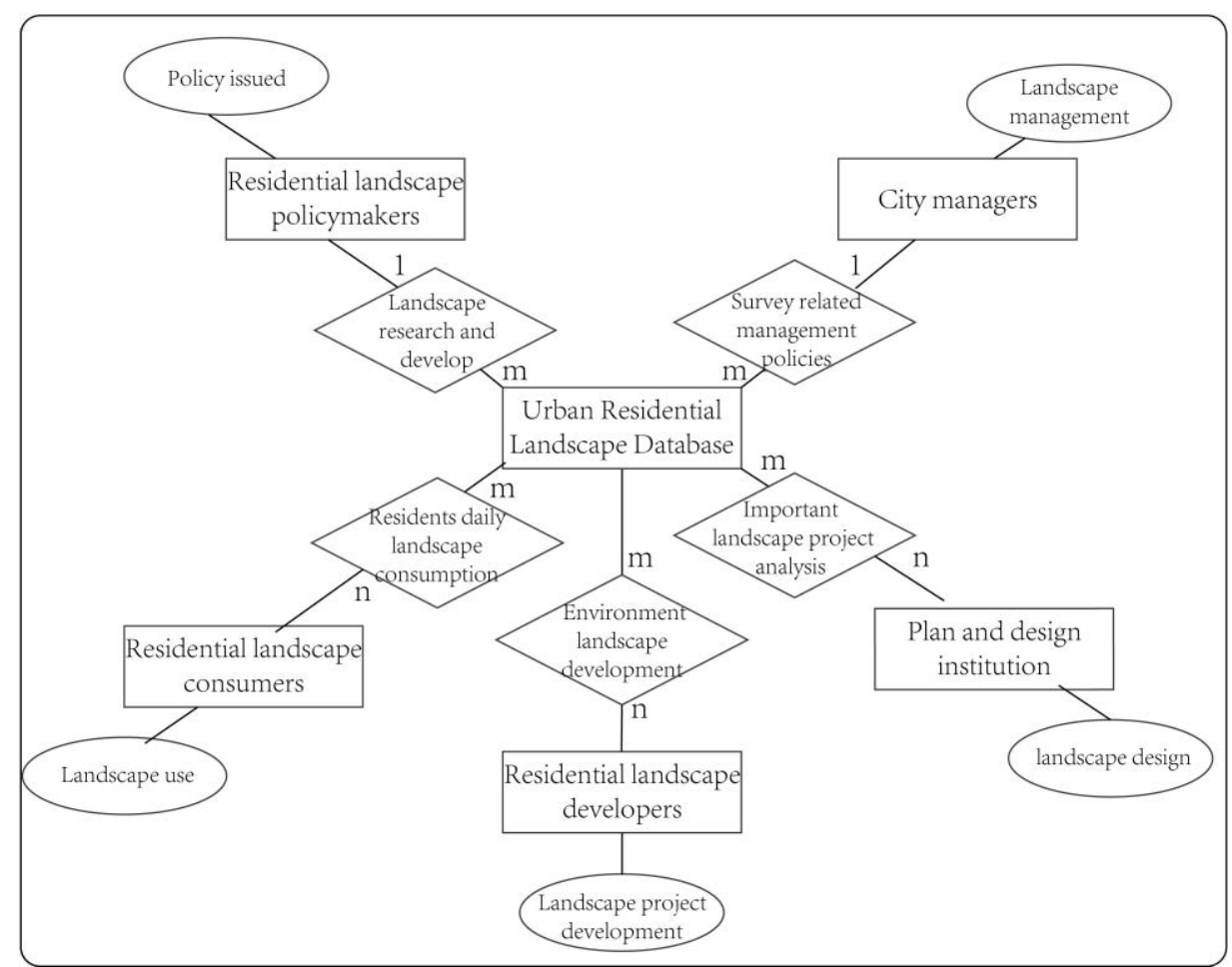

Figure 4. E-R Model of Urban Residential Landscape Database

It is an important database E-R model design tool, the constitute the basic elements of the entity type, properties, and contact. The database logic design to design E-R model, and then into relational model, urban residential landscape database E-R model as shown:

\subsection{Physical Design}

On the basis of logic design, physical design of database of data in the database on a physical device of storage structure and access method to carry on the design, ensure that the database things run efficiently. Physical design dependent on computer systems on the one hand, on the one hand, related to DBMS (database management system, including the field type, length, precision, and the DBMS data page size, response time, memory allocation, storage mode and index selection, etc.

Physical design of the software and hardware choice, of course, including the selection of the DBMS, should fully consider the application system of the satisfaction of demand, such as efficiency, capacity, security, DBMS, generally in Oracle, MS SQL server, DB2, MY SQL database and other well-known products choose according to need. Physical database construction process to classify the data, in particular to distinguish between dynamic and static data and multimedia data, large binary objects will be slow with new data and update the data monitoring, in order to achieve quick query, modify the data such as execution efficiency of the operation. 


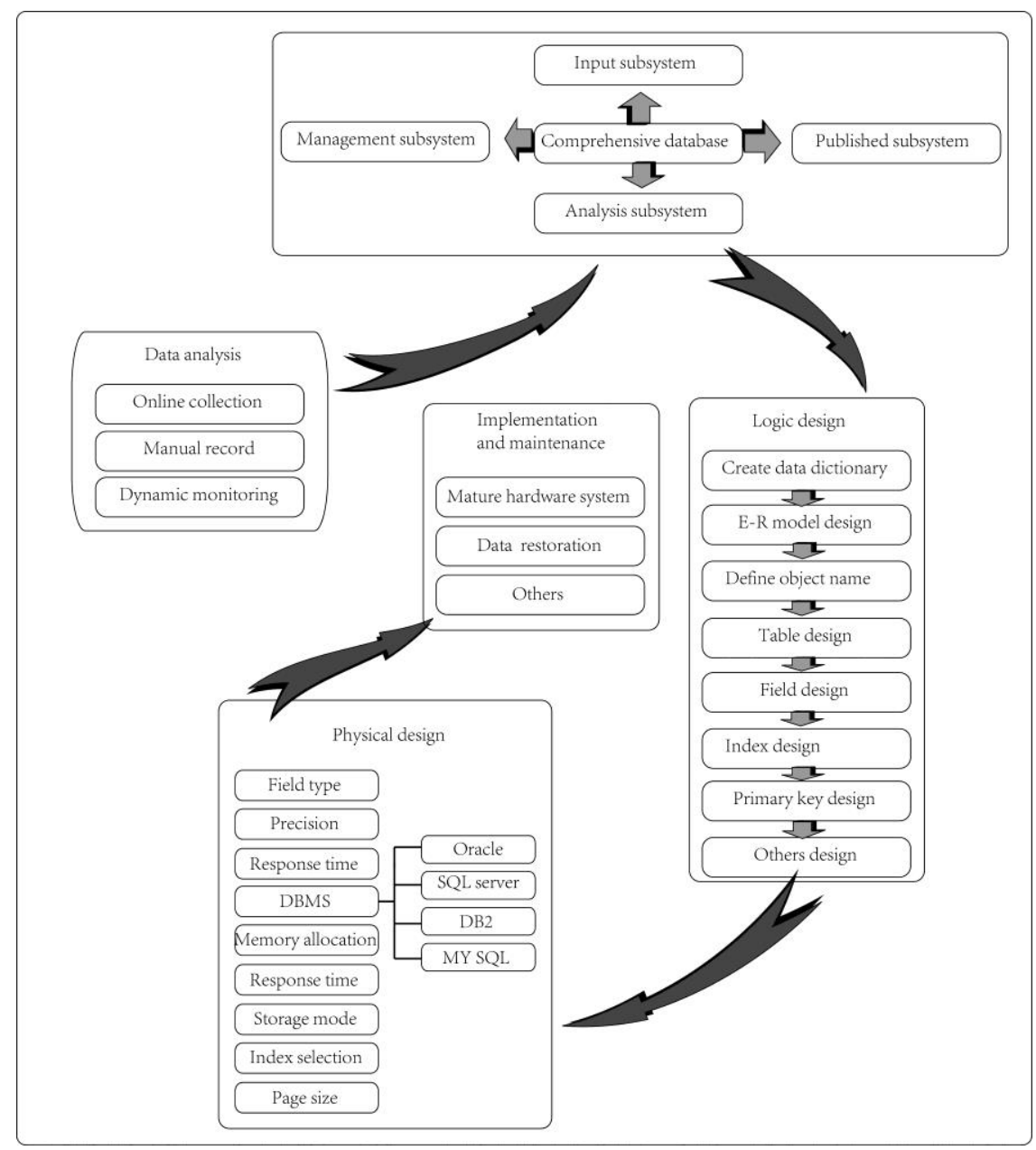

Figure 5. Model of Urban Residential Landscape Database

\subsection{Implementation and Maintenance}

In view of the residential landscape of contemporary environment, new urbanization construction as well as the importance of contemporary workplace experience, the reliability of the urban residential landscape database construction is very important, especially typical representative area, residential landscape engineering and household items, as well as in the old, old city transformation and other special landscape engineering collection, query and statistic analysis, the stability of the system is particularly important, but for security from the following aspects: first, choose a more mature and stable hardware system. Second, USES the high security of data backup and recovery system, so that the inevitable hardware failure occurs, realize the data recovery, to avoid greater losses.[7]

\section{The Residential Landscape Database Application based on DW Technique}

\subsection{Database Release with the Query}

The final purpose of database construction is a multi-user Web use, the urgency of the urban residential landscape construction and high quality requirements of city planning, urban landscape developer divide and consumer can timely grasp the residential environment landscape based on some demands of comprehensive and detailed data. 
Recommend choosing Adobe Dreamweaver site connections, release, maintenance and daily management work.

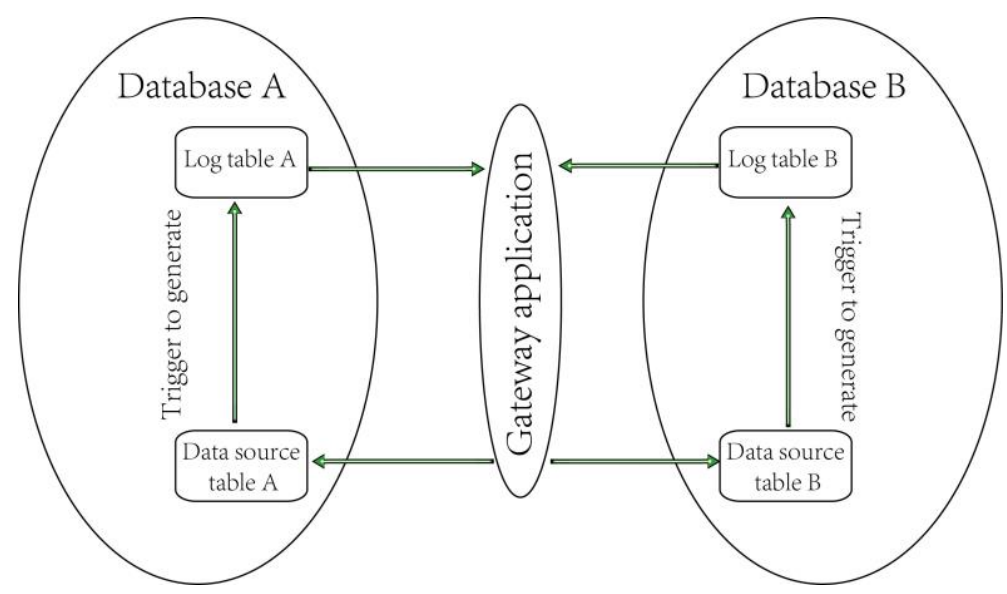

Figure 6. Process of Data Replication

The value of Web application is based on database user query and database updates, this determines the Web design must choose a dynamic Web technique. Dynamic Web page refers not to the dynamic effect, but the dynamic exchange of data between the user and the system. Made up of dynamic Web site usually based on the database technique, the Web has the points of static and dynamic, because can see the data updated in real time, and can finish the work is the database, using dynamic language implementation, and database interaction, so as to achieve the purpose of the data update, at the same time also greatly reduces the site maintenance work. Dynamic Web pages to the server for support, we usually meet in Html double-click can normal open at the end of the file, even if there is no server also won't affect our view pages, but the dynamic Web page is different, we need a server for support, at the same time, the server must also support this dynamic language to browse.

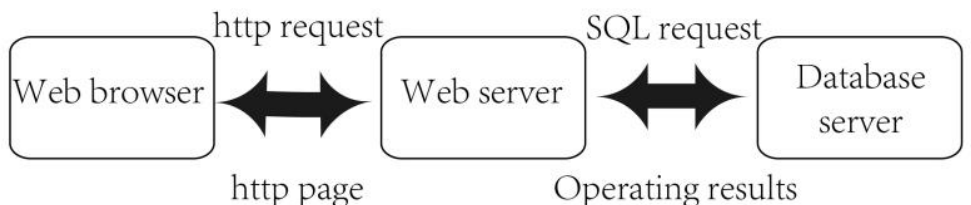

$$
\text { Client layer Application layer Data layer }
$$

Figure 7. Web Mode of Database Access

\subsection{Configuration of the Development Environment}

Application of the urban residential landscape database first need to match the development environment. According to the installation server can use Windows own IIS server, you can also use the more popular apache server, this need according to the use of dynamic language technique decision, asp is corresponding to the IIS, Php is apache. Then is the choice of the database, also need to choose according to their own dynamic languages, if you choose to use Asp dynamic language, need to Access database and the collocation of; If use Php and MySQL database need be collocation, after years of development experience, this paper recommends a combination of Php + MySQL, and 
then the dynamic combination of language and the database server. Development environment collocation is to realize the dynamic Web pages required steps, of course, the launch phase, Dreamweaver in such aspects as test, release, manage and use $\mathrm{Php}+$ MySQL collocation, has the good effect.

\section{Conclusion}

Big data era are raised informatization requirement on all of life. Construction urban residential landscape information database, it is an important basis that planning and design urban landscape residential environmental. For the aspect of urban residential landscape database construction. This paper discussed of Php + MYSQL with Dreamweaver techniques, to construction safe and efficient, reliable, relatively stable storage, operation and maintenance of the database, the experiences of expectation can speed up the construction of urban environment, especially in residential landscape with contemporary information the era of big data fusion, in order to achieve the informationization of urban residential landscape construction to provide some useful reference.

\section{Acknowledgements}

This research supported by Philosophy and Social Sciences Planning project of Heilongjiang Province (Project No.14E062).The author is director of Arts and technique Institute,Harbin University of Science and technique,research in the field cross of arts, techniques and social sciences.

\section{References}

[1] H. B. Li and J. G. Wu, "Use and misuse of landscape indices", Landscape Ecology, vol. 19, (2004), pp. 389-398.

[2] My SQL, Available:http://www.toadsoft.com, (2008).

[3] IBM, Corporation.Service-oriented Architecture Expands the Vision of Web Services, (2004).

[4] Z. Lix and H. He, R. C. Bu, "The adequacy of different landscape metrics for various landscape patterns", Pattern Recognition, vol. 38, (2005), pp. 232-263.

[5] J. Feng, Y. Sun and J. Lu, Net Ca:Aworld-wide CSCW authoring system for the WWW, CSCWID98, (1998).

[6] Z. Y. Sun, "One strategy of SQL syntax analysis and the achievement", Computer Application, China, vol. 27, (2007), pp.18-20

[7] ISO/IEC 18024-4, Information technique-SEDRIS language binding-Part4:C. 